\title{
Adicción a las redes sociales y sintomatología depresiva en universitarios
}

Carmen Padilla-Romero ${ }^{1}$ Juliana Ortega-Blas ${ }^{2}$

RESUMEN: El objetivo fue determinar la relación entre la adicción a redes sociales y la sintomatología depresiva en estudiantes de psicología de una Universidad de Lima Norte. Investigación correlacional de corte transversal. Para el análisis estadístico se usó Chi-cuadrado. Se emplearon los siguientes instrumentos: Cuestionario de adicción a las redes sociales (ARS) y el Inventario de depresión de Beck-Adaptado (BDI-IIA). Un 38.3\% de los encuestados reportó un nivel muy alto de adicción a las redes presentando un nivel severo de sintomatología depresiva. Se evidencia una correlación significativa entre las dimensiones de adicción a redes sociales y la sintomatología depresiva. Existe una relación directa en el aumento de los niveles de ambas variables en la muestra estudiada. Asimismo, cursar un ciclo de estudio inicial está asociado a la sintomatología depresiva severa. Las redes sociales podrían estar siendo utilizadas por este grupo poblacional para cubrir la baja autoestima, compensar la carencia de habilidades sociales y el aislamiento usualmente conectado a la sintomatología depresiva.

PALABRAS CLAVE: Adicción a redes sociales; Síntomas depresivos; Estudiantes.

Citar como: Padilla C, Ortega J. Adicción a las redes sociales y sintomatología depresiva en universitarios. CASUS. 2017;2(1):47-53.

${ }^{1}$ Centro de Terapias Integrales Edén. Licenciada en psicología. ${ }^{2}$ Colegio Parroquial Santa María de Jesús. Licenciada en psicología. ${ }^{1}$ Correo electrónico: padillaromero92@gmail.com Recibido: 08-01-2017 Aprobado: 15-04-2017 


\section{INTRODUCCIÓN}

La adicción a redes sociales y la sintomatología depresiva son temas investigados en la actualidad ya que están relacionados a problemas de salud mental (1). Al tema le añade relevancia la temprana edad de los usuarios de la red. Se estima que para el 2020 la depresión será la segunda causa de discapacidad a nivel mundial (2). Aunque ha sido ampliamente estudiada, sin embargo se continúa investigando cómo se comporta con variables del siglo XXI, era de la tecnología. Estudios realizados en universitarios evidencian asociación entre la adicción a la red social Facebook y la presencia de síntomas depresivos (3). En el Perú hay una prevalencia del 7\% de adicción a internet sobre todo en población joven y de educación superior (4). Respecto a la prevalencia nacional de episodio depresivo se encuentra entorno al $18,2 \%$ en adultos (5). Sin embargo, los síntomas depresivos en población universitaria ascienden respecto a la población en general, sobre todo en estudiantes pertenecientes a especialidades de ciencias de la salud (6).

El diagnóstico de adicción a internet se correlaciona con trastornos afectivos, con el acceso frecuente a las redes sociales y al ser varón (4). Así también, en el contexto universitario se señala que la inadecuada relación con el docente, el bajo rendimiento académico y la vulnerabilidad al estrés mantienen altos los índices de depresión (7).

Existen varias razones por las que los estudiantes universitarios son vulnerables a desarrollar adicción a las redes sociales. Comenzar la vida universitaria es un desafío, ya que implica ganar independencia e interactuar con nuevos compañeros para desarrollar su sentido de la identidad. Además en esta etapa se toman decisiones sobre diversos aspectos de la carrera, se debe lidiar con la presión de los estudios y del entorno social (8).

Hay una creciente popularidad del uso de las redes sociales entre los jóvenes. Esta se realiza a expensas de otras actividades de la vida cotidiana en particular aquellas que implican una interacción cara a cara. Sin embargo la investigación sobre el alcance de este fenómeno es insuficiente en jóvenes universitarios. Por otro lado, la relación entre la adicción a las redes sociales y la sintomatología depresiva no ha sido suficientemente explorada. Por lo tanto, este estudio ha tratado de explorar la relación entre la sintomatología depresiva y la adicción a las redes sociales. Por esta razón se propuso como objetivo analizar la relación entre la sintomatología depresiva y la adicción a las redes sociales en un grupo de jóvenes universitarios en Lima.

\section{MATERIALES Y MÉTODOS}

Estudio de tipo correlacional de corte transversal. La muestra estuvo conformada por 262 estudiantes universitarios seleccionados con un muestreo no probabilístico. Las variables principales fueron: adicción a redes sociales y sintomatología depresiva. La adicción a redes sociales se caracteriza por comportamientos repetitivos que resultan satisfactorios en primera instancia, pero una vez que se instalan como hábitos, generan estados de necesidad que son difícilmente controlados (9). La sintomatología depresiva se caracteriza por una tristeza patológica. Los síntomas varían entre la pérdida del interés en la mayor parte del día, sentimientos de culpa, irritabilidad, pérdida del apetito e ideación suicida (10). La adición a redes sociales es una variable cualitativa ordinal y se midió a través del Cuestionario de adicción a redes sociales (ARS). La misma comprende tres dimensiones: obsesión, falta de control personal y uso excesivo de las redes sociales categorizada en cuatro niveles: bajo, moderado, alto y muy alto que corresponden a la intensidad de la adicción. La sintomatología depresiva es una variable cualitativa ordinal y se midió a través del Inventario de depresión de Beck-Adaptado (BDI-IIA) adaptado al contexto nacional (11). Este inventario categoriza a la sintomatología depresiva en cuatro niveles: mínimo, leve, moderado y severo. En relación a las variables sociodemográficas se consideraron la edad: variable dicotómica (18- 20 años y 21 años a más); el sexo: nominal dicotómica y el ciclo de estudios que es ordinal.

Para el análisis de los datos se utilizó el paquete estadístico STATA. Se empleó el Chi-cuadrado en el análisis de las variables categóricas. Se consideró una significancia estadística menor a 0.05 . El presente estudio fue aprobado por el Comité de Ética de la Universidad Católica Sedes Sapientiae y los datos de los participantes fueron protegidos de acuerdo a los principios bioéticos.

\section{RESULTADOS}

Los resultados reportan un $52.3 \%$ de encuestados en el grupo etario de 18 a 20 años. Un $74.8 \%$ fueron mujeres. El tercer ciclo presenta la mayoría de 
estudiantes (23\%) seguido del quinto ciclo $(20.2 \%)$. El $22.9 \%$ de estudiantes presentó indicadores de adicción a redes sociales en un nivel muy alto.
Mientras que el $22.1 \%$ de la muestra reportó sintomatología depresiva en el nivel severo. El resto de los datos se puede observar en la tabla 1 .

Tabla 1. Descripción de las variables en universitarios

\begin{tabular}{lcc}
\hline & $\mathbf{n}$ & $\mathbf{( \% )}$ \\
\hline Grupo etario & & \\
I8- 20 años & 137 & 52.3 \\
21 años a más & 125 & 47.7 \\
Sexo & & \\
Mujer & & \\
Hombre & 196 & 74.8 \\
Ciclo de estudios & 66 & 25.2 \\
I & & \\
II & & 18.7 \\
III & 49 & 8.8 \\
V & 23.3 \\
VI & 23 & 14.5 \\
VIII & 61 & 20.2 \\
Adicción a las redes sociales & 38 & 5.3 \\
Muy alto & 53 & 9.2 \\
Alto & 14 & \\
Moderado & 24 & 22.9 \\
Bajo & & 23.7 \\
Sintomatología depresiva & 60 \\
Severo & 62 & 27.7 \\
Moderado & & \\
Leve & & \\
Mínimo & 68 & 22.1 \\
\hline
\end{tabular}

Se aprecia que entre los estudiantes del grupo etario de $18-20$ años $(26.3 \%)$, mujeres $(24.5 \%)$, que se encuentran en tercer ciclo (31.5\%) respectivamente presentan sintomatología depresiva severa. Existe una asociación significativa entre la adicción a redes sociales y la sintomatología depresiva $(\mathrm{p}=0.001)$. De igual manera se asocia el ciclo de estudio y los niveles de sintomatología depresiva $(\mathrm{p}=0.033)$. Los resultados muestran un $55.6 \%$ de estudiantes con un nivel bajo de adicción a las redes sociales que presentan un nivel de sintomatología depresiva mínima. Sin embargo, hay un $38.3 \%$ de encuestados con un nivel muy alto de adicción a las redes que presentan un nivel severo de sintomatología depresiva. También se observó una correlación significativa entre las dimensiones de adicción a redes sociales y la sintomatología depresiva. Hay un mayor porcentaje de estudiantes con un nivel muy alto en la dimensión falta de control personal con un nivel severo de sintomatología depresiva (44.1\%). El resto de los datos se puede observar en la tabla 2. 
Tabla 2. Asociación entre los niveles de sintomatología depresiva y la adicción a las redes sociales

\begin{tabular}{|c|c|c|c|c|c|}
\hline & $\begin{array}{c}\text { Mínimo } \\
\text { n(\%) }\end{array}$ & $\begin{array}{l}\text { Leve } \\
\mathrm{n}(\%)\end{array}$ & $\begin{array}{c}\text { Moderado } \\
\mathrm{n}(\%)\end{array}$ & $\begin{array}{c}\text { Severo } \\
n(\%)\end{array}$ & p-value \\
\hline \multicolumn{6}{|l|}{ Grupo etario } \\
\hline De 18 a 20 años & $42(30.7)$ & $32(23.4)$ & $27(19.7)$ & $36(26.3)$ & \multirow[t]{2}{*}{0.173} \\
\hline 21 años a más & $50(40.0)$ & $34(27.2)$ & $19(15.2)$ & $22(17.6)$ & \\
\hline \multicolumn{6}{|l|}{ Sexo } \\
\hline Mujer & $68(34.7)$ & $43(21.9)$ & $37(18.9)$ & $48(24.5)$ & \multirow{2}{*}{0.110} \\
\hline Hombre & $24(36.4)$ & $23(34.8)$ & $9(13.6)$ & $10(15.2)$ & \\
\hline \multicolumn{6}{|l|}{ Ciclo de estudios } \\
\hline I & $14(28.6)$ & $11(22.4)$ & $9(18.4)$ & $15(30.6)$ & \multirow[t]{7}{*}{0.033} \\
\hline II & $9(39.1)$ & $7(30.4)$ & $3(13.0)$ & $4(17.4)$ & \\
\hline III & $15(24.6)$ & $14(23.0)$ & $13(21.3)$ & $19(31.1)$ & \\
\hline IV & $8(21.1)$ & $11(28.9)$ & $10(26.3)$ & $9(23.7)$ & \\
\hline $\mathrm{V}$ & $22(41.5)$ & $16(30.2)$ & $8(15.1)$ & $7(13.2)$ & \\
\hline VI & $9(64.3)$ & $3(21.4)$ & $2(14.3)$ & - & \\
\hline VIII & $15(62.5)$ & $4(16.7)$ & $1(4.2)$ & $4(16.7)$ & \\
\hline \multicolumn{6}{|c|}{ Adicción a las redes sociales } \\
\hline Muy alto & $10(16.7)$ & $11(18.3)$ & $16(26.7)$ & $23(38.3)$ & \multirow{4}{*}{0.001} \\
\hline Alto & $20(32.3)$ & $18(29.0)$ & $8(12.9)$ & $16(25.8)$ & \\
\hline Moderado & $22(32.4)$ & $21(30.9)$ & $13(19.1)$ & $12(17.6)$ & \\
\hline Bajo & $40(55.6)$ & $16(22.2)$ & $9(12.5)$ & $7(9.7)$ & \\
\hline \multicolumn{6}{|c|}{ Obsesión por las redes sociales } \\
\hline Muy alto & $7(14.0)$ & $12(24.0)$ & $13(26.0)$ & $18(36.0)$ & \multirow{4}{*}{0.001} \\
\hline Alto & $11(22.4)$ & $15(30.6)$ & $8(16.3)$ & $15(30.6)$ & \\
\hline Moderado & $28(38.4)$ & $15(20.5)$ & $14(19.2)$ & $16(21.9)$ & \\
\hline Bajo & $46(51.1)$ & $24(26.7)$ & $11(12.2)$ & $9(10.0)$ & \\
\hline \multicolumn{6}{|c|}{ Falta de control personal } \\
\hline Muy alto & $3(8.8)$ & $9(26.5)$ & $7(20.6)$ & $15(44.1)$ & \multirow[t]{4}{*}{0.001} \\
\hline Alto & $15(28.3)$ & $10(18.9)$ & $12(22.6)$ & $16(30.2)$ & \\
\hline Moderado & $15(29.4)$ & $16(31.4)$ & $10(19.6)$ & $10(19.6)$ & \\
\hline Bajo & $59(47.6)$ & $31(25.0)$ & $17(13.7)$ & $17(13.7)$ & \\
\hline \multicolumn{6}{|c|}{ Uso excesivo de las redes } \\
\hline Muy alto & $27(26.0)$ & $23(22.1)$ & 19(18.3) & $35(33.7)$ & \multirow[t]{4}{*}{0.001} \\
\hline Alto & $12(27.3)$ & $14(31.8)$ & $9(20.5)$ & $9(20.5)$ & \\
\hline Moderado & $30(39.5)$ & 19(25.0) & $16(21.1)$ & $11(14.5)$ & \\
\hline Bajo & $23(60.5)$ & $10(26.3)$ & $2(5.3)$ & $3(7.9)$ & \\
\hline
\end{tabular}




\section{DISCUSIÓN}

Los resultados revelan que los niveles de sintomatología depresiva se asocian con los niveles de adicción a redes sociales, así también, se relacionan el ciclo de estudios con la sintomatología depresiva.

Se observó que el nivel severo de sintomatología depresiva se asocia a un nivel muy alto de adicción a redes sociales. Este hallazgo es corroborado por otros estudios $(1,14)$. Una posible explicación podría relacionarse a la presencia de algunos indicadores depresivos como una autoestima baja caracterizada por presentar una percepción negativa de sí, escasos recursos de interacción social y el aislamiento social. Estos elementos conllevarían a la adicción a redes sociales (12). Por otro lado, el anonimato que ofrecen las redes sociales favorecería la construcción de una identidad ficticia para lograr compensar el estado emocional depresivo por el cual atravesaría el usuario (13). Asimismo, el estado afectivo depresivo favorece el acceso a internet en un intento de disminuir los síntomas depresivos con el fin de mejorar su relación social a través de este medio virtual (14). Se observa que las redes sociales son utilizadas como vía para mostrar estados de contenido depresivo, que al ser reforzados por otros contactos de manera intermitente, mantendría la conducta de publicar en la red (15).

Existe asociación entre la dimensión falta de control personal de la adicción a redes sociales y el nivel severo de sintomatología depresiva. Un modelo reporta que el criterio escaso control sobre sí forma parte de la adicción a las nuevas tecnologías como las redes sociales que, a su vez, se relaciona con el desajuste emocional (16). Igualmente la falta de control es crucial para determinar adicción a sustancias. Se encuentran pruebas que respaldan esta asociación que implica a su vez la falta de control (17).

Se halló que la sintomatología depresiva severa se asocia con el cursar ciclos iniciales de la carrera universitaria. Otros estudios corroboran estos resultados indicando que los estudiantes que cursan los primeros ciclos pueden presentar mayores índices de trastornos afectivos, debido a los diferentes cambios que atraviesan en el contexto universitario $(18,19)$. Con el paso de los años, en este ambiente, van disminuyendo los niveles de ansiedad o depresión debido al proceso de adaptación (20)
Entre las principales limitaciones del estudio se señala que no fueron incluidas variables que pueden estar relacionadas con la adicción a redes sociales y a la sintomatología depresiva como el tipo de familia de proveniencia, la frecuencia de uso de redes, el tiempo dedicado y la tipología de la red social. A pesar de esta limitación los principales hallazgos concuerdan con estudios publicados $(1,3,13)$.

\section{CONCLUSIONES}

Se evidencia una relación directa en el aumento de los niveles de ambas variables en la muestra estudiada. Asimismo, cursar un ciclo de estudio inicial está asociado a la sintomatología depresiva severa. Las redes sociales podrían estar siendo utilizadas por este grupo poblacional para cubrir la baja autoestima, compensar la carencia de habilidades sociales y el aislamiento usualmente conectado a la sintomatología depresiva.

Se recomienda seguir investigando los factores asociados a ambas variables en esta población, ya que es vulnerable debido a las características propias del entorno. A su vez se propone realizar programas preventivos promocionales que potencien una adecuada autoestima y las habilidades sociales. Los mismos deben informar adecuadamente de las consecuencias negativas de la adicción a las redes proponiendo intervenciones que modulen el impulso al uso adictivo de las mismas. 


\section{REFERENCIAS BIBLIOGRÁFICAS}

1. Pantic I, Damjanovic A, Todorovic J, Topalovic D, Bojovic D, Risti S, et al. Association between online social networking and depression in high school students: Behavioral Physiology viewpoint. Psychiatria Danubina. 2012;24(1):90-93.

2. Organización Mundial de la Salud (OMS). Clasificación de los trastornos mentales y del comportamiento (CIE-10). Con Glosario y Criterios Diagnósticos de Investigación. España, Madrid: Editorial médica panamericana; 2004.

3. Herrera MF, Pacheco MP, Palomar J, Zavala AD. Adicción a Facebook Relacionada con la Baja Autoestima, la Depresión y la Falta de Habilidades Sociales. Psicología Iberoamerica. 2010;18(1):6-18.

4. Cruzado L, Matos L y Kendallfolmer R. Adicción a internet: Perfil clínico y epidemiológico de pacientes hospitalizados en un Instituto Nacional de Salud Mental. Rev Med Hered. 2006;17(4):196-205. http://www.scielo.org.pe/pdf/rmh/v1 7n4/v17n4ao2.pdf.

5. Instituto Nacional de Salud Mental. Estudio epidemiologia de salud mental en Lima Metropolitana y Callao- Replicación 2012. Informe General. 2013. Anales de Salud Mental, (29), Suplemento 1.Lima.

6. Pereyra R, Ocampo J, Silva V, Vélez E, da Costa AD, Toro LM, et al. Prevalencia y factores asociados con síntomas depresivos en estudiantes de ciencias de la salud de una Universidad privada de Lima, Perú 2010. Rev Perú Med Exp Salud Pública. 2010;27(4):520-26.

7. Fouilloux M, Barragán P, Ortiz L, Jaimes A, Urrutia ME, Guevara G. Síntomas depresivos y rendimiento escolar en estudiantes de Medicina. Salud mental. 2013;36(1):59-65.
8. Niemz K, Griffiths M, y Banyard P. Prevalence of pathological Internet use among university students and correlations with self-esteem, the General Health Questionnaire (GHQ), and disinhibition. CyberPsychology \& Behavior. 2005;8(6):562-570.

9. Escurra M y Salas E. Construcción y validación del cuestionario de adicción a redes sociales (ARS). Liberabit. 2014;20(1):73-91. Recuperado de

$\mathrm{http}: / /$ ateneo.unmsm.edu.pe/ateneo/ha ndle/123456789/3472.

10. Asociación Americana de Psiquiatría. Guía de consulta de los criterios diagnósticos del DSM 5. (5ta ed.). Estados Unidos: Arlington; 2013.

11. Carranza RF. Propiedades psicométricas del Inventario de depresión de Beck universitarios de Lima. Rev. Psicol Trujillo. $2013 ; 15$ ( 2 ) : $152-158$. http://www.ucv.edu.pe/sites/publicaci ones/psicologia/2013-2.pdf.

12. Jiménez AL. y Pantoja V. Autoestima y Relaciones Interpersonales en Sujetos Adictos, a Internet. Psicología-Segunda Época. 2007;26(1):78-89.

13. Young K. Rodgers R. The Relationship Between Depression and Internet Addiction. Cyber Psychology \& Behavior. 1998;1(1):25-28.

14. Tsai C, Sunny S, y Lin A. Internet Addiction of Adolescents in Taiwan: An Interview Study. Cyber Psychology \& Behavior. 2003:6(6):649-652.

15. Moreno MA, Jelenchick, LA, Egan KG, Cox E, Young H, Gannon, KE. Feeling Badon Facebook: Depression disclosures by college students on a Social Networking Site. Depression and Anxiety. 2011;28(6):447-455. doi:10.1002/da.20805.
16. Senormanci Ö, Komkan R, y Zihni M. Internet Addiction and Its Cognitive Behavioral Therapy. Santandard and Innovative Strategies in Cognitive Behavior Therapy. 2012.171-190.

17. Gómez DT, Gutierrez M, y Londoño SP. Depresión y consumo de sustancias psicoactivas en adolescentes en la ciudad de Bogotá. Psychologia. Avances de la disciplina. 2013;7(1):45-51.

18. Osada J, Rojas M, Rosales C, Vega J. Sintomatología ansiosa y depresiva en estudiantes de medicina. Rev Neuropsiquiatr. 2010;73:15-19.

19. Rodas P, Santa Cruz G, Vargas H. Frecuencia de trastornos mentales en alumnos del tercer año de la Facultad de Medicina de una universidad privada de Lima-2006. Rev Med Hered. 2009;20:70-76.

20. Galli A. Prevalencia de trastornos psicopatológicos en alumnos de psicología. Revista de psiquiatría y salud mental Hermilio Valdizan. 2005;6(1):55-66. 


\section{Addiction to social networks and depressive symptomatology in university students}

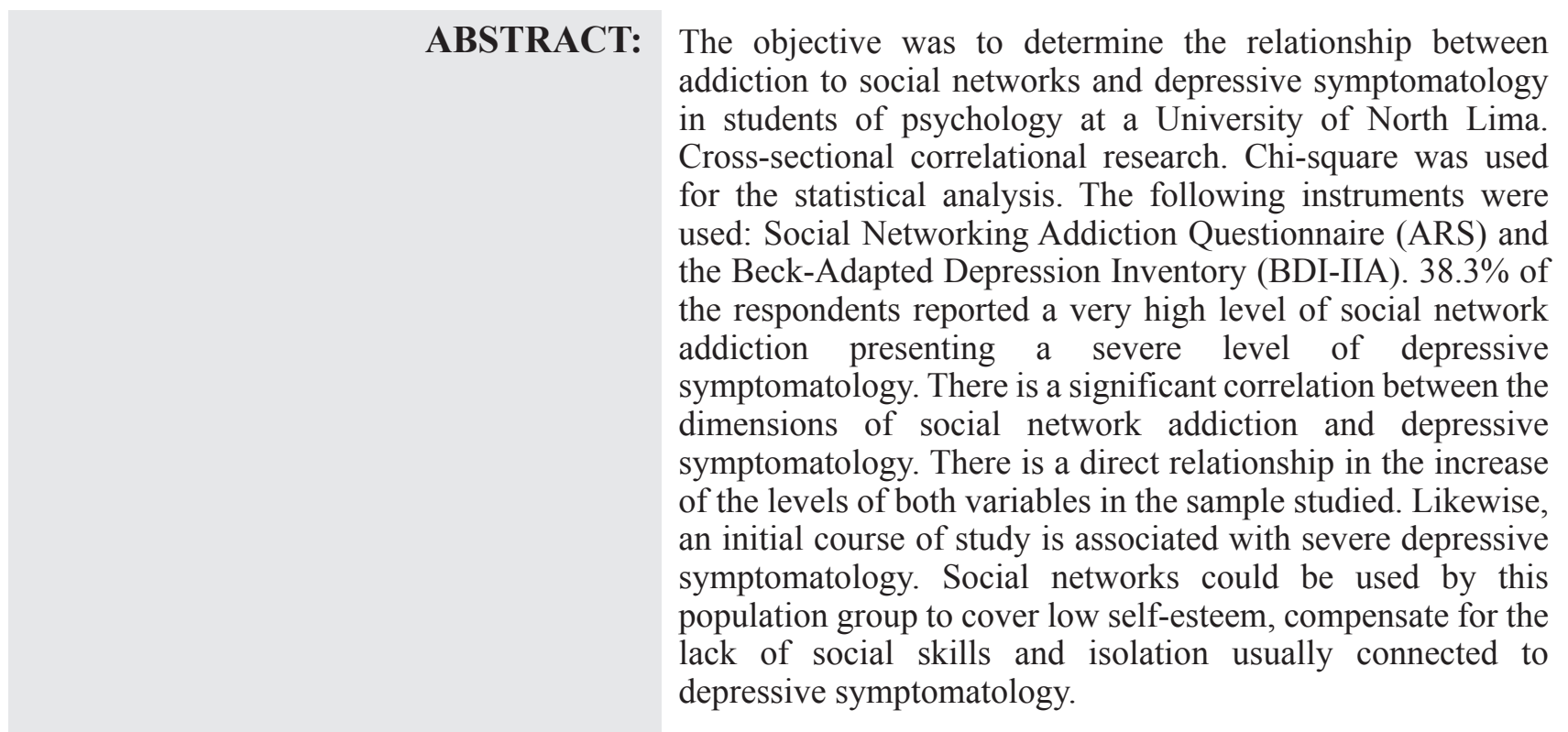

KEY WORDS: Addiction to social networks and internet; Depression; Students. 